\title{
WATER RECOVERY WITH THE HEAT MELT COMPACTOR IN A MICROGRAVITY ENVIRONMENT
}

\author{
Eric L. Golliher ${ }^{1 *}$, Jonathan Goo ${ }^{2}$, John Fisher ${ }^{3}$ \\ ${ }^{1}$ NASA Glenn Research Center, Cleveland, Ohio, 44135 \\ ${ }^{2}$ Case Western Reserve University, Cleveland, Ohio, 44106 \\ ${ }^{3}$ NASA Ames Research Center, Moffett Field, California 94035-1000
}

\begin{abstract}
The Heat Melt Compactor is a proposed utility that will compact astronaut trash, extract the water for eventual re-use, and form dry square tiles that can be used as additional ionizing radiation shields for future human deep space missions. The Heat Melt Compactor has been under development by a consortium of NASA centers. The downstream portion of the device is planned to recover a small amount of water while in a microgravity environment. Drop tower low gravity testing was performed to assess the effect of small particles on a capillary-based water/air separation device proposed for the water recovery portion of the Heat Melt Compactor.
\end{abstract}

KEY WORDS: Life Support, Heat Transfer, Condensation

\section{INTRODUCTION}

In order to complete long-term human deep space missions, many resources are required to support humans and logistics operations of the spacecraft. Due to the isolation of the spacecraft from earth, and the impracticality of periodic resupply from earth, solutions must be found for providing all the resources. The ideal solution to provide those resources is to reuse and repurpose the resources. Among the efforts to develop such technology to reuse resources is the heat melt compactor (HMC) project. The HMC separates water from trash used by astronauts by evaporating the water within the compactor and then condensing it downstream from the compactor [1]. The water is then condensed and separated from the gas and residual vapor in a dedicated condenser and dedicated separator, or in a combined condenser/separator. The water is then directed to a water re-processing unit for chemical purification and processing, which makes the water useable. This paper discusses one aspect of the separator unit: the operation in microgravity with particles that may possibly degrade a capillary-based separator. The main challenge in separating the liquids from the gases arises because of the lack of gravity while in space. On Earth, the greater density of liquids compared to gases causes the liquids to sink to the bottom of a container while the gases rise. However, in space, where there is no gravity to separate the two phases alternate forces must be applied such as capillary action. The capillary separator and HMC have been investigated by other researchers [2], [3]. A concern for using a capillary separator is contamination with small solid particles that may impede the capillary-driven liquid flow through the separator and cause the separator to be inefficient or non-functional. This paper reports on the observation of silicone oil mixed with solid glass beads inside a wedge while in microgravity conditions. The testing device is called an angular liquid bridge (ALB) vessel. The ALB was an experiment performed on board the MIR space station by astronauts in order to examine capillary flow in microgravity. The ALB hardware was an astronaut training unit and brought out of storage for this drop tower testing. Microgravity 
conditions were simulated by dropping the ALB while it was secured to drop rig in the 2.2 second drop tower at the Glenn Research Center. The liquid behavior was observed using an HD camera, which was also secured to the drop rig. The video data was further analyzed using Sony Vegas Pro, a commercial video editing program, and Spotlight, an image analysis and tracking software previously developed at NASA GRC.

\section{BACKGROUND}

Capillary action is the ability of a liquid to flow along a solid due to a combination of intermolecular forces. There is cohesion within the liquid as well as adhesion between the liquid and the solid. These forces can be observed on the earth by looking at the surface of a cup of coffee as it curves upward near the edge of the cup, or as water wicks into a paper towel. Capillary action occurs as a liquid seeks to find an equilibrium state where it contains the least amount of energy by balancing the energy from the pressure within itself, the pressure coming from its surroundings, and its surface tension energy. To minimize the energy of the liquid, the total surface area will be minimized, given the constraints of contact angle, surface tension, and gravity. The relationship between the pressures at the interface of the liquid and the surface tension is also related to the curvature of the liquid by equation 1 , where $\mathrm{R}_{1}$ and $\mathrm{R}_{2}$ are orthogonal radii along the surface and $\gamma$ is the surface tension [4].

$\Delta p=\gamma\left(\frac{1}{R_{1}}+\frac{1}{R_{2}}\right)$

The relationship between the contact angle of a liquid and the surface tension between the three different interfaces is given by equation 2, where $\theta$ is the contact angle between the liquid and surface, and $\gamma_{L G}, \gamma_{S G}$, $\gamma_{S L}$ are the liquid to gas, solid to gas, and solid to liquid surface tension values [4].

$\gamma_{L G} \cos (\theta)=\gamma_{S G}-\gamma_{S L}$

Normally while on the earth, in $1-\mathrm{g}$, gravity is the dominant force that establishes an equilibrium surface shape, while capillary action has only a minor effect. In space, where there is no gravity, capillary action becomes the dominant force acting on liquids, making a capillary separator a potential method of liquid-air separation while in space. Figure 1 shows a conceptual illustration of a surface at equilibrium in $1-\mathrm{g}$ which suddenly experiences 0 -g. This describes the free surface time history of the experiments reported in this paper. By introducing various types and amounts of solid particles, and by observing the change in speed of the capillary-driven flow, we can make some preliminary conclusions about the effect of solid particles on capillary-driven flow in a wedge.

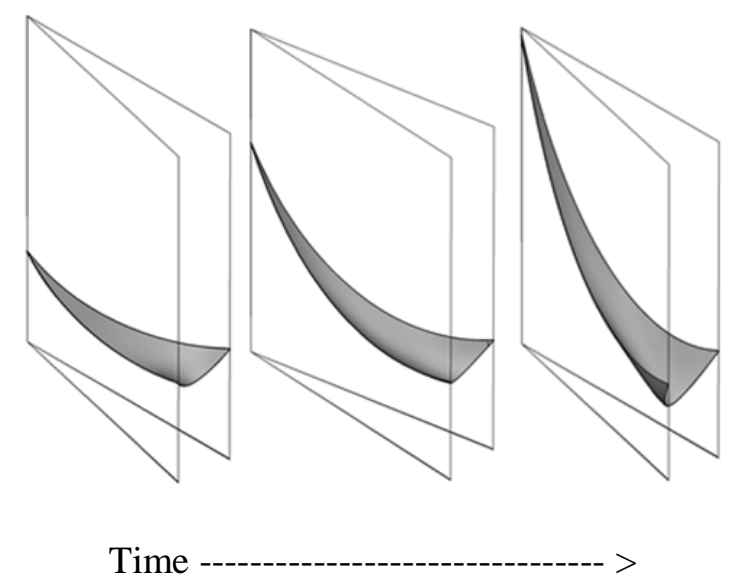

Figure 1 Movement of a Capillary Free Surface in a Wedge 


\section{EXPERIMENTAL STUDIES}

In order to evaluate the behavior of a liquid in a wedge in microgravity, an Angular Liquid Bridge (ALB) vessel was used. As shown in Figure 2, the ALB vessel holds liquid between two flat polycarbonate plates that meet at an angle. Initially, gravity pools the liquid at the bottom of the ALB. The corner formed by the two plates causes the liquid to wick up slightly into this corner. In 1-g, this wicking is minor. Upon the sudden change from $1 \mathrm{-g}$ to $0 \mathrm{-g}$, as occurs in the 2.2 second drop tower, the liquid that had pooled at the bottom of the ALB begins to move rather quickly into the corner. At equilibrium in microgravity, the liquid would completely shift into the corner. However, the 2.2 second duration of the drop does not allow the liquid to reach an equilibrium condition. This is not needed, however, since the goal is to observe the movement of the liquid and how that movement changes when different types and amount of small particles are added. The liquid used in the ALB is silicone oil. Silicone oil has a low viscosity and contact angle. The combination of viscosity and contact angle make it ideal for observing significant free surface movement during the 2.2 second experiment duration. The contact angle of silicone oil is affected little by dissolved impurities, allowing consistency between experimental trials. Glass beads were used to simulate the small particles that might be found in the fluids extracted from the heat melt compactor. Two types of beads were used, borosilicate beads $3 \mathrm{~mm}$ in diameter with a density of 2.23 $\mathrm{g} / \mathrm{ml}$ and soda-lime silica beads approximately $0.5 \mathrm{~mm}$ in diameter with a density of $2.5 \mathrm{~g} / \mathrm{ml}$.
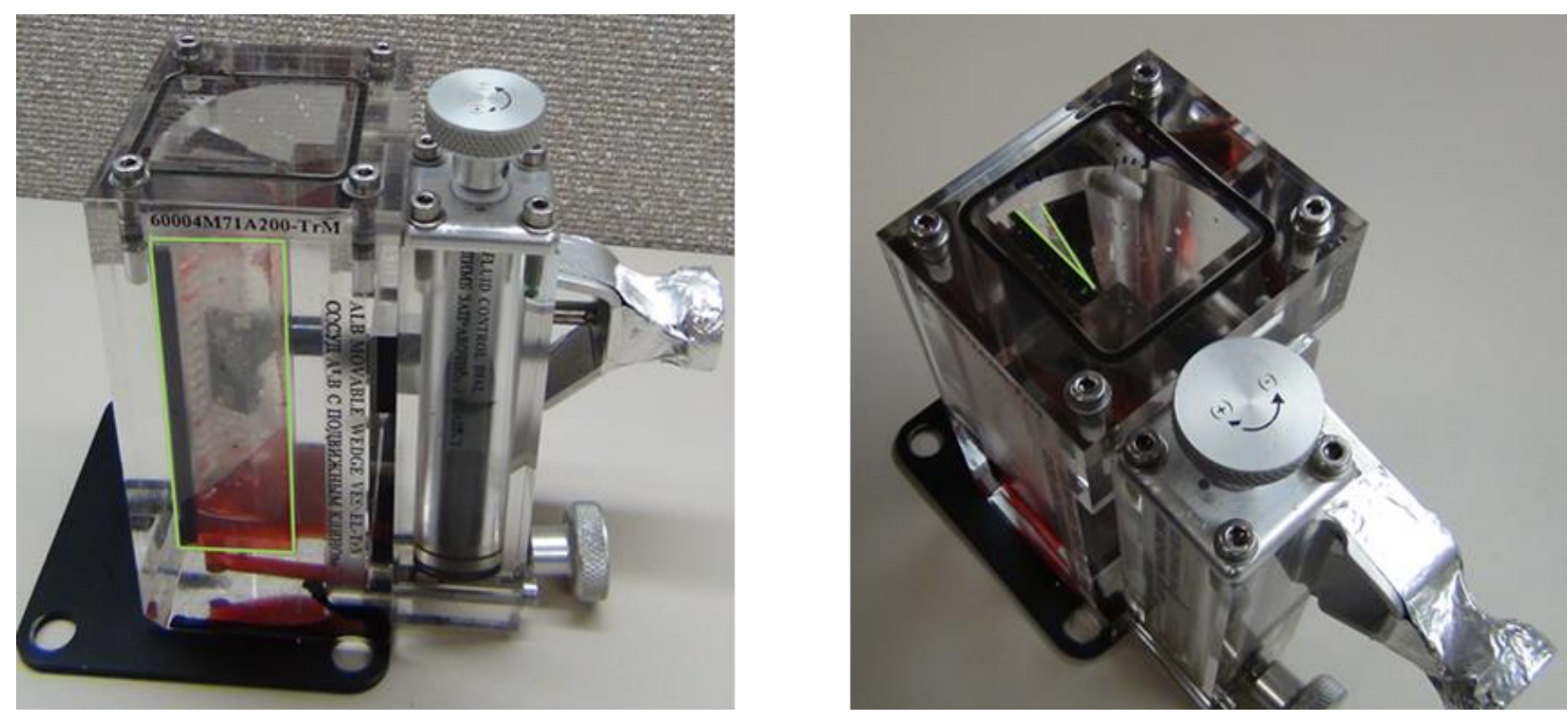

Figure 2 Angular Liquid Bridge Vessel Front and Top Views

To simulate the low gravity conditions of space, the ALB was secured to a drop rig and dropped in the 2.2 second drop tower located at the NASA Glenn Research Center as shown in Figure 3. The drop rig used is an aluminum rectangular frame where the ALB and a camera can be secured. The drop rig is also placed inside of a drag shield, which is a closed metal container that will fall slightly slower than the rig within itself because it experiences air drag while shielding the rig inside from the drag. The drop tower is approximately 24 meters in height and is able to drop small experiments in drop rigs from the top in order to put the experiment into free fall. While the rig is in free fall, ALB experiences microgravity. 

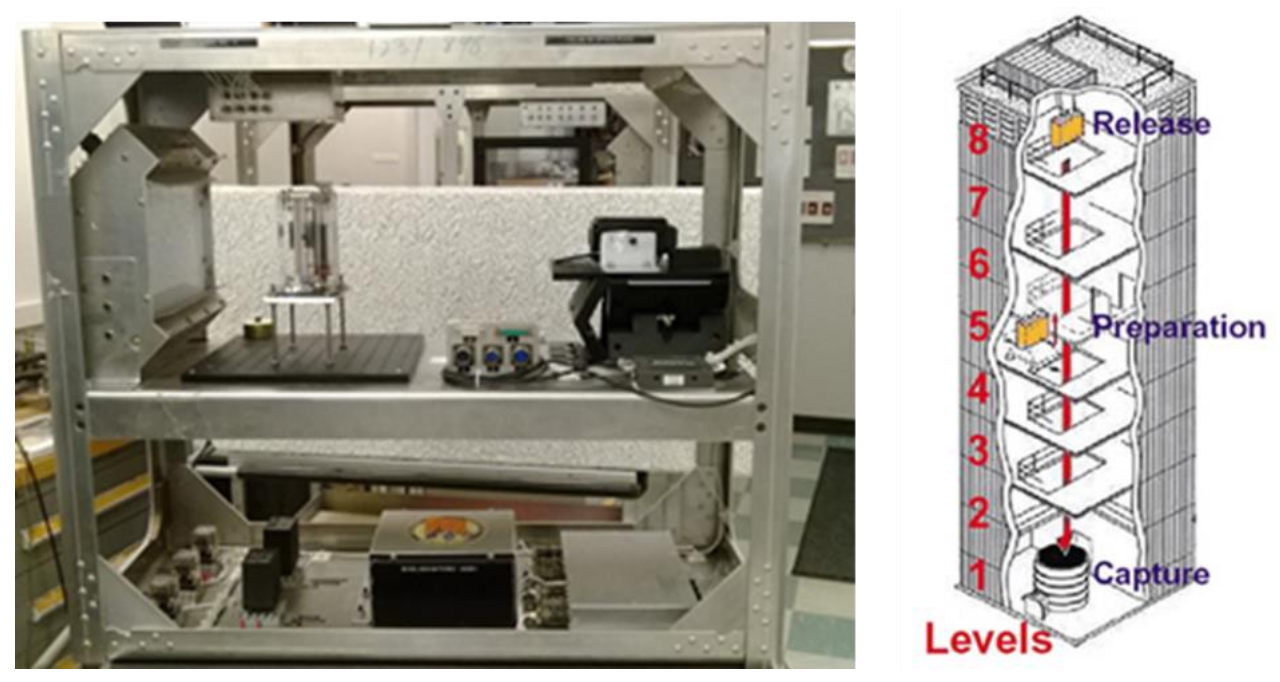

Figure 3 Drop Tower Rig and Illustration of the Glenn Research Center 2 Second Drop Tower

The amount of time experienced in free fall can be confirmed using the following equation comparing time to position, at constant acceleration as described in equations 3 and 4, where $g$ is the acceleration due to gravity, $t$ is the duration of the drop, and $h$ is the tower height.

$h=\frac{1}{2} g t^{2}$

$t=\sqrt{\frac{2 h}{g}}$

At the bottom of the tower is an airbag to catch and decelerate the drag shield and drop rig. This systems protects everything by reducing the shock that the equipment experiences. The camera used to record the behavior of the liquid during the experiment was a high definition Sony Camcorder, model HDR-CX260V/W. The camera recorded at 30 frames per second with a resolution of 1920x1080/60p. Within the ALB the same amount of silicone oil was used for every drop and was reused for each drop. The internal plate angle was constant at $13 \pm 0.5^{\circ}$. For each drop the number of beads, the type of bead, the orientation and placement of the beads, and the orientation of the ALB were varied. During the initial experiments, $3 \mathrm{~mm}$ diameter borosilicate beads were used. These beads were relatively large and did not affect the flow, possibly because the major portion of the free surface was nearer to the corner vertex and not affected by the presence of the bead. In other trials, smaller $0.5 \mathrm{~mm}$ diameter soda-lime silicate beads were used. These intercepted the free surface near the vertex and did affect the flow. The orientation of the beads was also varied to see how beads placed at different positions would affect the flow. In order to ensure that the beads would stay near the vertex of the corner, the orientation of the ALB was tilted so the beads would remain aligned at their initial placement. The ALB vertical axis for these later trials was tilted to an angle of $81.3^{\circ}$ as opposed to the baseline trials of $90^{\circ}$. Once the video data was collected, the video was edited for observation using Sony Vegas Pro 11.0 and then further analyzed using the Spotlight image analysis and tracking software. A total of 19 successful trials were conducted in the drop tower. Below is a description of each trial.

\section{Trial 1}

There were no beads. The ALB was placed level to the ground.

\section{Trial 2}

Repeated Trial 1 and changed the field of view to maximize the resolution of video by zooming in closer to the region where the liquid moves. This was the baseline to compare to Trials 3 through 9 . 


\section{Trial 3}

One 3 millimeter bead was placed as close to the vertex of the corner as possible and submerged in the oil. The lighting was also changed from a front light to a backlight to improve the visibility of the bead.

\section{Trial 4}

Four $3 \mathrm{~mm}$ beads were placed linearly along bottom, submerged.

\section{Trial 5}

Eight $3 \mathrm{~mm}$ beads were placed making two layers of beads from the bottom, submerged.

\section{Trial 6}

Fourteen $3 \mathrm{~mm}$ beads were placed making three layers of beads from the bottom, submerged.

\section{Trial 7}

Fourteen $3 \mathrm{~mm}$ beads were placed making two layers from bottom submerged in the oil and 5 were lined along the vertex not submerged.

\section{Trial 8}

Fifteen $3 \mathrm{~mm}$ beads were placed making three layers of beads from the bottom, submerged, and two beads were not submerged.

\section{Trial 9}

Thirty $3 \mathrm{~mm}$ beads were placed making three layers of beads submerged and eight beads not submerged.

\section{Trial 10}

The trial was without any beads and the ALB base was tilted $8.7^{\circ}$. This trial is the base test for all trials 11-19.

\section{Trial 11}

Thirty-five $0.5 \mathrm{~mm}$ beads were scattered throughout ALB.

\section{Trial 12}

0.5 grams of $0.5 \mathrm{~mm}$ beads were scattered throughout ALB.

\section{Trial 13}

This trial repeated trial 12. After dropping the ALB in trial 12 many beads that started out not submerged in the oil fell to the bottom of the ALB and were submerged.

\section{Trial 14}

$0.5 \mathrm{~g}$ of $0.5 \mathrm{~mm}$ beads were scattered throughout the ALB but some were stacked inside the wedge. In previous trials the plates were not touching the bottom of the ALB and beads would slide out of the wedge. For this trial the plates were pushed down to prevent beads from sliding out of the wedge.

\section{Trial 15}

$4 \mathrm{~g}$ of $0.5 \mathrm{~mm}$ beads were stacked within wedge past the oil-air interface. The oil-air interface shifted due to the beads. This caused the initial position of the interface to be higher than previous trials.

\section{Trial 16}

$2 \mathrm{~g}$ of $0.5 \mathrm{~mm}$ beads stacked slightly past the oil-air interface in the base test.

\section{Trial 17}

$0.5 \mathrm{~mm}$ beads were placed till slightly below oil-air interface. The oil-air interface returned to the same position as the base test. 


\section{Trial 18}

$0.5 \mathrm{~mm}$ beads were stacked till the beads were at the same position as the interface.

\section{Trial 19}

$0.5 \mathrm{~mm}$ beads were stacked just below oil-air interface.

\section{EXPERIMENTAL RESULTS}

Various bead configurations resulted in different rates of liquid movement. If the liquid movement was similar to the baseline case which had no beads, that particular bead configuration was judged as one that would not affect a capillary-type separator. At time $=0$, the oil was pooled near the bottom of the $\mathrm{V}$-shaped groove, the groove being oriented vertically. Some of the oil is wicked into the corner. At the moment of drop rig release, the experiment experiences microgravity. With no gravity force to pull the oil downward, the surface tension moves the oil into the V-shaped corner. The oil viscosity is too high to for the free surface to reach its equilibrium value, but sufficient low to observe significant free surface movement during the drop.

Figure 4 displays the position of the liquid at time zero and after 2 seconds of being in microgravity for the baseline case of no beads and the closer viewpoint, trial 2.

Trials 3 through 9 were conducted using the $3 \mathrm{~mm}$ beads. As the oil free surface reached the beads, some secondary wicking occurred in the spaces between the beads. The oil wicking along the beads allowed the oil to move faster at a very local level on the order of the bead length scale. Once the oil level passed the beads, the oil would continue flowing slower. Overall, there was little change in the gross flowrate in trials 3 through 9 when comparing the results those from the base test.

In trials 11 through 14 , the first trials conducted using the $0.5 \mathrm{~mm}$ beds, the oil continued to behave the same, flowing at the similar rate as in the base test, trial 10. However, trials 15 through 19 showed significant change in the behavior of the liquid. The liquid either did not move or moved at a slower rate.
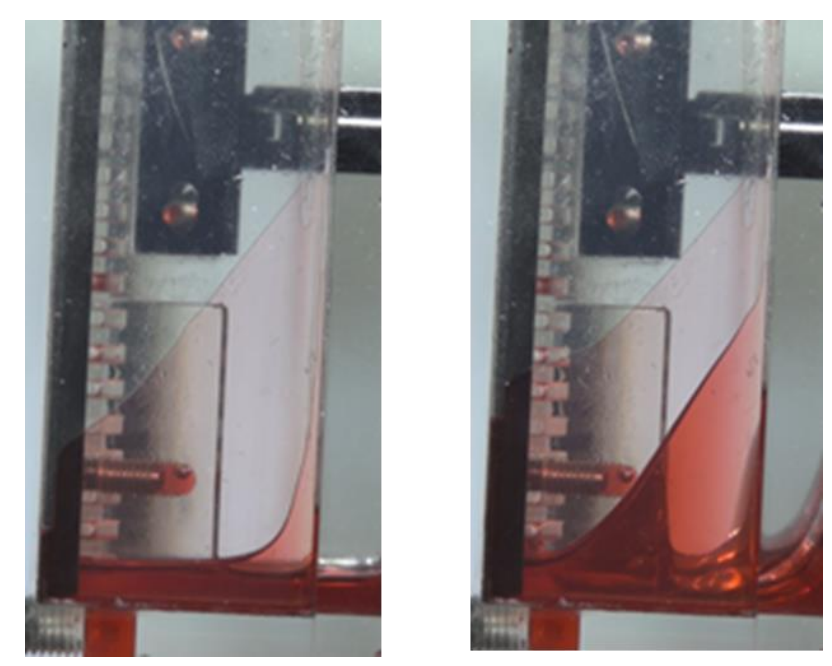

Figure 4 Showing Fluid Movement in V-Shaped Wedge with Time (Time Lapse is 2 Seconds)

Figure 5 shows the baseline (no beads) free surface movement as analyzed by the Spotlight software discussed earlier. There are three lines which display the position of the free surface liquid interface outline at 0,1 , and 2 seconds. The origin of each graph corresponds to the top right corner of the ALB vessel (as the pixels increase for the axis labeled "Pixels in y-direction" the position is moving downward and as the pixels 
increase along the axis labeled "Pixels in x-direction" the position is moving in the left direction). As can be seen in Figure 5, there is significant movement of the free surface during the drop. Figure 6 shows the free surface movement for Trial 5 which was a typical trial for all of the large bead results, trials 3 through 9. As can be seen in Figure 6, there is little change from the baseline final free surface profile compared to the baseline shown in Figure 5. Figure 7 shows the free surface movement for Trial 15 which is representative of the small bead results that caused interference with the flow.

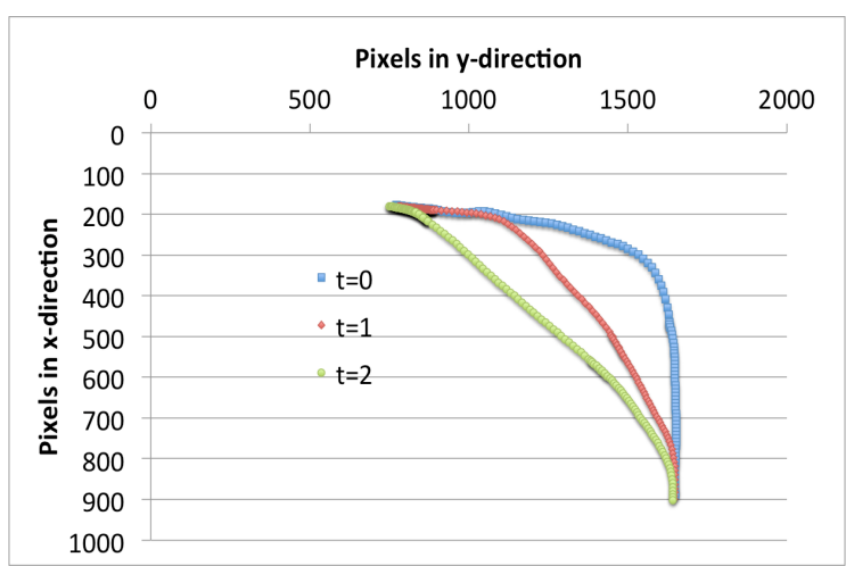

Figure 5. Baseline Results Showing the Movement of the Free Surface with Time

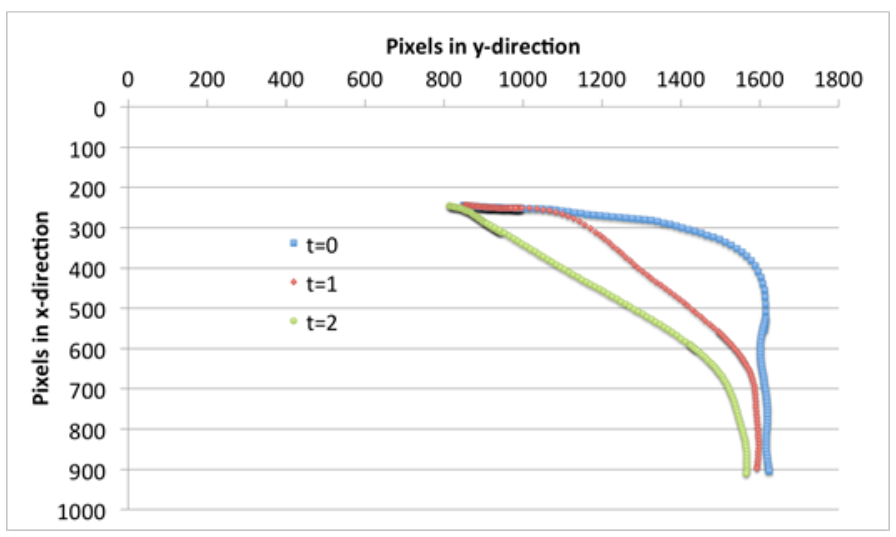

Figure 6. Trial 5 Results Showing Similar Movement of the Free Surface with Time with the Baseline Trial

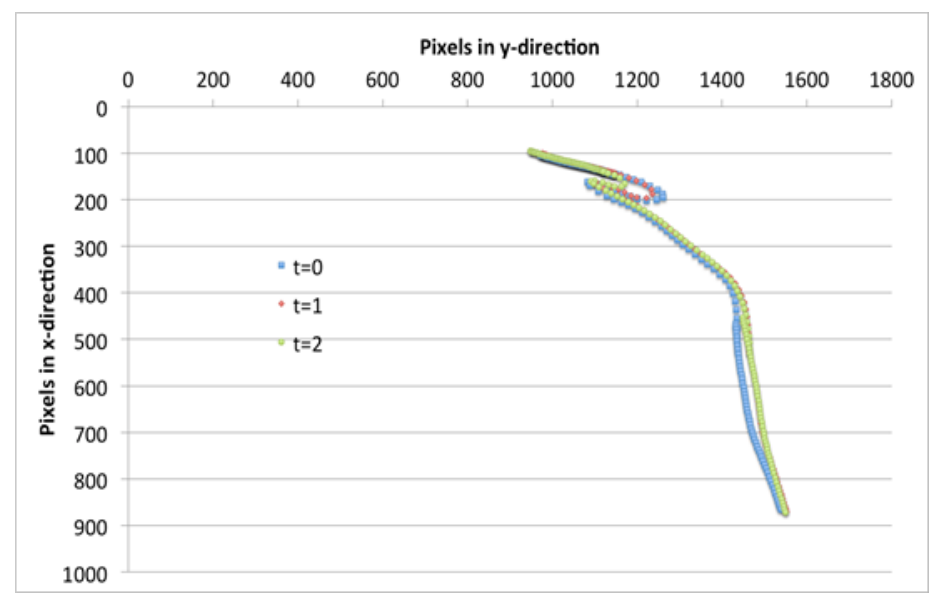

Figure 7. Trial 15 Results Showing Little Movement of the Free Surface with Time 


\section{CONCLUSIONS}

Based on these experimental results, a capillary separator should be a feasible method for separating the condensed water from the gases and vapor during the operation of the heat melt compactor (HMC), provided that the free surface is not interrupted by the particles. The flow appears to be unaffected with small amounts of solid particle contamination in the liquid. When the solid contaminates are relatively large and cannot reach into the corner to change the free surface, the flow is not affected. When the particle size is very small and the particles are totally submerged such that they do not interfere with the free surface, the flow is also not affected. When the number of small particles is large and when the particles do interfere with the free surface, the flow is adversely affected.

\section{FUTURE WORK}

To define potential future work in this area, we can identify are some areas of potential improvement for the experimentation test matrix. The interior corner angle was constant at $13 \pm 0.5^{\circ}$, because the ALB could not be adjusted to a smaller angle. Another issue was the errant wicking of oil into other ALB corners. This was consistent between trials and therefore did not affect a change in flow with each trial. Also, the solids shifting out of place slowed the process of reconfiguring the bead placement for each new trial. Future investigations could benefit greatly from newly designed hardware that would allow improvement of the test matrix.

\section{ACKNOWLEDGMENT}

The authors would like to thank Eric Neumann, Dan Gedeon, John Doehne, and Alex Camargo for helping to conduct the experiments in the drop tower. The authors would also like to thank John McQuillen and Jay Owens for their assistance in setting up the experiment. The authors would also like to thank Mark Hickman who was critical to the scheduling of the drop tower resources for this research.

\section{REFERENCES}

[1] Golliher, E. L., Gotti, D. J., Rymut, J. E., Nguyen, B. K., Owens, J. C., Pace, G. S., Fisher, J. W., Hong, A. E., "Evaporative Heat Transfer Mechanisms within a Heat Melt Compactor", 43rd International Conference on Environmental Systems; 14-18 Jul. 2013; Vail, CO; United States

[2] Weislogel, M. M., Thomas, E., Graf, J., Systems And Methods For Separating A Multiphase Fluid, U S Patent 7905946, 2008

[3] Fisher, J.W., Pace, G.S., Wignarajah, K., Harris, L.C., Delzeit, L.D., Alba, R., “An Assessment of The Water Extraction Capabilities of the Heat Melt Compactor", 43rd International Conference on Environmental Systems; 14-18 Jul. 2013; Vail, CO; United States

[4] de Gennes, P.G.; Brochard-Wyart, F.;Quere, D., Capillarity and Wetting Phenomena: Drops, Bubbles, Pearls, Waves, Springer Science and Business Media, 2004. 